\title{
Análise retrospectiva dos pacientes infectados por RSV na unidade de transplante de medula óssea
}

\author{
RSV infection after allogeneic hematopoietic stem cell transplantation (HSCT): \\ Analysis of 59 patients transplanted in a single institution
}

Flavia Z. Piazera

Sergio C. Fortier ${ }^{1}$

Juliane Morando

Carmem M. S. Bonfim ${ }^{2}$

Rodney F. Silva ${ }^{3}$

Clovis A. Cunha

José Zanis Neto

\begin{abstract}
O vírus sincicial respiratório (RSV) é considerado uma causa importante de morbimortalidade em pacientes submetidos ao transplante de células-tronco hematopoéticas (TCTH). Mesmo com o uso da ribavirina inalatória (RI), as taxas de mortalidade são de $30 \%$ a $40 \%$. O objetivo deste trabalho foi analisar o perfil dos pacientes infectados pelo RSV e a eficácia do tratamento com RI. Realizou-se uma análise retrospectiva de 59 pacientes submetidos ao TCTH com infecção confirmada pelo RSV (métodos de IFI ou PCR) entre 02/1991 e 02/2008. A RI foi administrada por 12 horas, na dose de $5 \mathrm{~g}$ diluída $200 \mathrm{ml}$ de água destilada, por cinco dias. Quinze pacientes apresentaram infecções (TRI) do trato respiratório inferior e 44 pacientes apresentaram infecções (TRS) de vias aéreas superiores. No grupo tratado $(n=50)$, quarenta apresentaram infecções no TRS versus dez TRI; no grupo não tratado, quatro TRS versus cinco TRI. Foram constatados vinte óbitos (33,8\%), sendo que 13 desses pacientes (65\% dos óbitos) tiveram suas mortes relacionadas ao RSV. Dentre estes, nove pacientes foram a óbito antes da instituição da RI como terapia padrão. A sobrevida global (SG) de todos os pacientes foi de 8,3 meses, sendo 66\% para o grupo que utilizou RI versus $11,1 \%$ no grupo não tratado $(p=0,001)$. No entanto, a $S G$ foi inferior nos pacientes que apresentaram infecções no TRI (37,5\%) quando comparadas às infecções do TRS $(65,1 \%), p=0,007$. No modelo de regressão de Cox, a única variável independente encontrada foi o tratamento com RI $(p=0,001)$. Rev. Bras. Hematol. Hemoter.
\end{abstract}

Palavras-chave: Virus sincicial respiratório; transplante de medula óssea.

\section{Introdução}

O vírus sincicial respiratório pertence à família Paramyxoviridae, é RNA vírus, ${ }^{1}$ sendo uma das infecções respiratórias mais comuns na infância. Muitas crianças imunocompetentes são infectadas por volta dos 2 anos de idade, desenvolvendo anticorpos neutralizadores. ${ }^{2-6} \mathrm{~A}$ transmissão do RSV se faz através de secreções de vias aéreas e fomites. ${ }^{7}$ A mortalidade em crianças imunocompetentes é cerca de $0,5 \%{ }^{7}$ As taxas de mortalidade em pacientes imunocomprometidos, incluindo pacientes pós-TCTH, pode variar de $80 \%$ a $100 \%$ nos pacientes com infecções do trato respiratório inferior não tratados ou tratados já com sinais de injúria respiratória.

Nos pacientes pós-TCTH, a progressão de infecções do trato respiratório superior para trato respiratório inferior

\footnotetext{
${ }^{I}$ Residente em Serviço de Transplante de Medula Óssea - Hospital de Clínicas da Universidade Federal do Paraná - Curitiba-PR.

${ }_{3}^{2}$ Médica do Serviço de Transplante Pediátrico do HC-UFPR - Curitiba-PR.

${ }^{3}$ Pneumologista do Serviço de TMO do HC-UFPR - Curitiba-PR.

${ }_{5}^{4}$ Infectologista do Serviço do TMO do HC-UFPR - Curitiba-PR.

${ }^{5}$ Chefe do Serviço de Transplante de Medula Óssea do HC-UFPR - Curitiba-PR.
}

Hospital de Clínicas da Universidade Federal do Paraná - Curitiba-PR.

Correspondência: Flavia Zattar Piazera

Rua Nilo Cairo, 524, apto 204 - Centro

80.060-050 - Curitiba-PR - Brasil

E-mail:fpiazera@terra.com.br

Doi: 
pode variar de $40 \%$ a $60 \%{ }^{9-12} \mathrm{O}$ tratamento preemptivo com ribavirina inalatória pode prevenir a progressão de infecção respiratória alta dos pacientes pós-TCTH para pneumonia em torno de $70 \% .{ }^{9-13}$ Porém, há controvérsias na literatura sobre sua utilização diante de seus efeitos mielotóxicos, custo dispendioso, efeitos adversos nos profissionais de saúde que manipulam este medicamento e necessidade de hospitalização para sua administração. ${ }^{14}$

Não está estabelecido o tempo ideal de tratamento, bem como a dose e seu intervalo de administração.

Historicamente, em nosso serviço, as infecções por RSV foram uma grande causa de morbimortalidade nos pacientes transplantados. Simultamenamente, nove pacientes foram a óbito no ano de 1990 por quadro de infecção respiratória alta com progressão para infecção respiratória baixa (pneumonia), evoluindo rapidamente com insuficiência respiratória aguda e óbito. Neste período, a identificação do RSV era rudimentar, resultando no atraso no diagnóstico, bem como na instituição da terapêutica. Por isso o interesse neste assunto, tanto do ponto de vista terapêutico quanto do ponto de vista epidemiológico e infectológico.

\section{Casuística e Método}

Realizamos uma análise retrospectiva de 59 pacientes que foram submetidos ao TCTH com infecção confirmada pelo RSV (métodos de IFI ou PCR) entre 02/1991 e 02/2008.

Todos os pacientes admitidos na unidade de transplante de medula óssea para condicionamento pré-TMO, durante transplante, e os pacientes com acompanhamento ambulatorial com infecção pelo RSV foram incluídos nesta análise.

Após confirmação da infecção pelo RSV (após ano de 2003), a tomografia computadorizada de tórax foi utilizada de rotina em todos os pacientes para avaliação da presença de pneumonite associado a radiografia de tórax.

Devido à experiência trágica do nosso serviço perante os casos de RSV, é rotina realizar tratamento de todos os pacientes com infecção pelo RSV (vias aéreas superiores ou inferiores).

A pesquisa de RSV foi realizada em todos os pacientes com sintomas respiratórios altos e baixos. $\mathrm{O}$ aspirado de nasofaringe, lavado broncoalveolar e coleta de coriza foram os procedimentos realizados para identificação do vírus.

De fevereiro de 1991 a fevereiro de 2004, foi utilizado método de imunofluorescência indireta (IFI) para pesquisa do RSV (único método de deteç̧ão disponível nesta instituição). A partir de fevereiro de 2004, iniciou-se pesquisa pelo PCR- RT (reação de cadeia da polimerase em tempo real) qualitativo. Após coleta da amostra das secreções de vias aéreas, o resultado da positividade é fornecido por volta de 24 a 36 horas após coleta.

A RI foi administrada por 12 horas (início às 18 horas até as 6 horas do dia seguinte), na dose de $5 \mathrm{~g}$ diluída em $200 \mathrm{ml}$ de água destilada, por cinco dias, após confirmação da positividade do PCR para RSV. Não realizamos a administração de imunoglobulina intravenosa nos pacientes submetidos à terapia com ribavirina inalatória.

A análise estatística foi conduzida pelo software SPSS versão 15 , com $p<0,001$ como significante. $O$ método de Kaplan-Meier foi utilizado para cálculo de sobrevida global nos pacientes tratados com ribavirina inalatória e a regressão de Cox para cálculo da análise uni e multivariada das variáveis simples.

\section{Resultados}

A mediana de idade dos 59 pacientes transplantados com infecção pelo RSV foi de 19,9 anos (variação 2,8-54,02). Quanto às características gerais, 37 pacientes eram do sexo masculino e 22 do sexo feminino (Tabela 1 ).

Quanto ao tipo de doador, 48 pacientes realizaram transplante aparentado; destes, sete receberam célulastronco periféricas e 41 receberam medula óssea. Onze pacientes, um transplante não aparentado, sendo que três receberam sangue de cordão umbilical e os demais de medula óssea. Quanto aos métodos de detecção do RSV, 51 pacientes realizaram IFI (a maioria teve a infecção antes de 2004) e oito pacientes realizaram identificação pelo método do PCR qualitativo. Quinze pacientes apresentaram infecções (TRI) no trato respiratório inferior $(25,4 \%)$, tendo como achados clínicos: coriza, obstrução nasal, tosse seca, estertores bolhosos e sibilos na ausculta pulmonar. Quarenta e quatro pacientes apresentaram infecções (TRS) de vias aéreas superiores (74,5\%), demonstrando ao exame clínico: coriza, obstrução nasal, tosse seca, odinofagia. A mediana de dias pós-TCTH para o diagnóstico do RSV foi de 17 dias (variação 1- 435 dias). As doenças de base que determinaram a indicação do TCTH foram: anemia aplástica severa (AAS) - 18 pacientes; leucemia mieloide crônica (LMC) - 17; leucemia mieloide aguda (LMA) - 7; leucemia linfoide aguda (LLA) 3; mielodisplasia (MDS) - 6; anemia de Fanconi - 1; outras -

Tabela1. Características dos pacientes pós- TCTH com infecção por RSV

\begin{tabular}{lcc}
\hline \multicolumn{1}{c}{ Características } & $N^{\circ}$ pacientes & $(\%)$ \\
\hline Sexo & & \\
$\quad$ Feminino & 22 & 37,2 \\
Masculino & 37 & 62,7 \\
Tipo de doador & & \\
$\quad$ Aparentado & 48 & 81,3 \\
$\quad$ Não aparentado & 11 & 18,7 \\
Método de detecção & & \\
$\quad$ PCR & 9 & 13,5 \\
Imunofluorescência & 50 & 86,4 \\
Doença & & \\
$\quad$ Benigna & 26 & 44 \\
Maligna & 33 & 55,9 \\
\hline
\end{tabular}


7 pacientes. O tratamento com ribavirina inalatória foi realizado em cinquenta pacientes, sendo quarenta com infecções no TRS e dez com infecção no TRI. Os pacientes que não foram tratados foram quatro com infecção do TRS e cinco do TRI. Foram constatados vinte óbitos $(33,8 \%)$ do total de pacientes analisados $(\mathrm{n}=59)$, sendo que 13 desses pacientes $(65 \%$ dos óbitos) tiveram suas mortes relacionadas ao RSV. Todos esses 13 evoluíram com insuficiência respiratória aguda e necessidade de ventilação mecânica, sendo que nove foram a óbito antes da adoção da RI como terapia padrão nesta Instituição. Os três pacientes restantes iniciaram a terapia com ribavirina inalatória. No entanto, não terminaram o tratamento devido à progressão de insuficiência respiratória aguda. Todos os vinte pacientes que foram a óbito encontravam-se ainda sem sinais de pega do enxerto quando adquiriram infecção pelo RSV. A sobrevida global (SG) de todos os pacientes foi de 8,3 meses, sendo $66 \%$ para o grupo que utilizou RI versus $11,1 \%$ no grupo não tratado, $p=0.001$. No entanto, a $S G$ foi inferior nos pacientes que apresentaram infecções no TRI $(37,5 \%)$ quando comparadas às infecções do TRS $(65,1 \%), \mathrm{p}=0,007$. O tipo de doador, método de identificação do vírus, o tempo de diagnóstico ou a doença de base não foram estatisticamente significativos para SG. No modelo de regressão de Cox, a única variável independente encontrada foi o tratamento com $\mathrm{RI}(\mathrm{p}=0,001)$.

\section{Discussão}

O tratamento para infecções respiratórias pelo RSV na literatura mundial permanece ainda controverso: tempo de tratamento, dose adequada, forma de infecção (vias aéreas superiores ou inferiores), via de administração da ribavirina (inalatória ou endovenosa).

Muitos autores questionam o benefício do tratamento das infecções pelo RSV, ${ }^{11,12,13,16,20,22}$ pois não há publicações comparando populações homogêneas com as diversas opções e formas terapêuticas. As recomendações terapêuticas atuais estão baseadas em série de casos ou experiências de vários serviços.

Este trabalho identificou diminuição da mortalidade nos pacientes tratados com ribavirina inalatória quando comparados aos não tratados $(\mathrm{p}<0.001)$. Todos os pacientes não tratados evoluíram a óbito em decorrência de pneumonia associada à insuficiência respiratória aguda pelo RSV. Assim, sugerimos o benefício do tratamento precoce com ribavirina instituído nos pacientes com infecções no TRS, evitando progressão para pneumonia e óbito.

Nossos dados são semelhantes aos de Adams et al. ${ }^{20}$ que realizaram o tratamento preemptivo em 29 crianças pósTCTH por infecções assintomáticas pelo RSV. Todas foram tratadas com ribavirina inalatória, sendo que nenhuma apresentou progressão para pneumonia pelo RSV.

Entretanto, contraria o trabalho de Machado et al., ${ }^{22}$ no qual 18 pacientes pós-TMO apresentaram infecções do
TRS e não realizaram tratamento com ribavirina inalatória. Desses pacientes, $55 \%(\mathrm{n}=10)$ desenvolveram pneumonia pelo RSV.

A infecção do trato respiratório inferior foi um fator de diminuição da sobrevida global dos pacientes, comparado aos dados do trabalho do Khanna et al. ${ }^{16}$ A mortalidade foi mais expressiva durante a fase pré-pega do enxerto (mediana do diagnóstico no $\mathrm{D}+17$ pós-TMO), provavelmente devido à vulnerabilidade imunológica. Neste estudo, a mortalidade relacionada à infecção pelo RSV foi superior aos estudos publicados na literatura. ${ }^{12,13,15,16,20,22}$ A mortalidade global foi de $33 \%$, sendo $65 \%$ dos óbitos relacionados ao RSV.

Porém, a mortalidade diminuiu nos últimos anos, provavelmente devido a métodos diagnósticos mais sensíveis, rápida instituição da terapia mesmo nos casos de infecções leves e padronização das medidas terapêuticas no serviço.

Não tivemos nenhuma suspensão do tratamento por efeitos adversos da ribavirina, bem como retardo da pega do enxerto em decorrência de seu uso, semelhante aos trabalhos mais recentemente publicados. ${ }^{16,15,17}$

Vários trabalhos citam uma evolução rápida para infecções TRI e aumento da mortalidade nos pacientes submetidos a transplantes não aparentados. Porém, a única característica independente com impacto positivo na sobrevida global foi utilização da ribavirina inalatória, $\mathrm{p}=0,001$.

Em conclusão, a infecção pelo RSV desempenha um impacto significativo na morbimortalidade dos pacientes submetidos ao TCTH. Este estudo ressalta o benefício da ribavirina inalatória no tratamento de infecções pelo RSV: com diminuição da mortalidade dos pacientes que receberam tratamento, sem efeitos adversos. Estudos multicêntricos são necessários para definição de critérios de tratamento e nas intervenções baseadas na ribavirina são mais adequados neste grupo de pacientes.

\footnotetext{
Abstract

Respiratory syncytial virus (RSV) causes significant mortality in patients submitted to SCT. Despite the use of ribavirin aerosols $(R A)$, mortality rates are still between 30 and $40 \%$ in many centers. The objective of this study was to analyze the clinical course and outcome of 59 patients who developed RSV infections after SCT in a single institution. In this retrospective analysis, the diagnosis of RSV infection was confirmed in 59 patients submitted to HSCT. RA was administrated during 12 hours at a dose of $5 \mathrm{~g}$ diluted in 200 $m L$ of distillated water, for 5 days. Fifteen patients presented with upper respiratory tract (URT) infection and 44 patients presented with lower respiratory tract (LRT) infection. In the group of patients who received $R A(n=50), 40$ had $U R T$ infections and 10 had LRT infections. In patients who did not receive $R A, 3$ had URT infections and 6 had LRT infections. Twenty patients died (33.8\%) with the main cause of death of 13 patients being RSV infection (all these patients required mechanical ventilation). Nine patients died before
} 
$R A$ therapy became standard treatment for RSV (before 1992). The overall survival of patients treated with $R A$ was $66 \%$. However, the overall survival was lower in patients who had LRT infections (37.5\%) compared with those who had URT infections $(67.5 \%$ $p=0.007)$. In the multivariate analysis, only the use of $R A$ affected overall survival $(p=0.001)$. Rev. Bras. Hematol. Hemoter.

Key words: Respiratory syncytial virus; allogeneic hematopoietic stem cell transplantation.

\section{Referências Bibliográficas}

1. Domachowske JB, Rosenberg HF. Respiratory syncytial virus infection: immune response, immunopathogenesis, and treatment. Clin Microbiol Rev. 1999;12(2):298-309.

2. Hall CB. Respiratory syncytial virus and parainfluenza virus. New Engl J Med. 2001;344:1917-28.

3. Glezen WP, Taber LH, Frank AL, Kasel JA. Risk of primary infection and reinfection with respiratory syncytial virus. Am J Dis Child. 1986;140(6):543-6.

4. Falsey AR, Walsh EE. Respiratory syncytial virus infection in adults. Clin Microbiol Rev. 2000;13(3):371-84.

5. Hall CB, Walsh EE, Long CE, Schnabel KC. Immunity to and frequency of reinfection with respiratory syncytial virus. J Infect Dis. 1991;163(4):693-8.

6. Hall CB, Geiman JM, Biggar R, Kotok DI, Hogan PM, Douglas GR Jr. Respiratory syncytial virus infections within families. N Engl J Med. 1976;294(8):414-9.

7. Prober CG, Sullender WM. Advances in prevention of respiratory syncytial virus infections. J Pediatr. 1999;135(5):546-58.

8. Harrington RD, Hooton TM, Hackman RC, Storch GA, Osborne $\mathrm{B}$, Gleaves CA, et al. An outbreak of respiratory syncytial virus in a bone marrow transplant center. J Infect Dis. 1992; 165 (6): 987-93.

9. Abdallah A, Rowland KE, Schepetiuk SK, To LB, Bardy P. An outbreak of respiratory syncytial virus infection in a bone marrow transplant unit: effect on engraftment and outcome of pneumonia without specific antiviral treatment. Bone Marrow Transplant. 2003;32(2):195-203

10. Whimbey E, Champlin RE, Englund JA, Mirza NQ, Piedra PA, Goodrich JM, et al. Combination therapy with aerosolized ribavirin and intravenous immunoglobulin for respiratory syncytial virus disease in adult bone marrow transplant recipients. Bone Marrow Transplant. 1995;16(3):393-9.

11. Couch RB, Englund JA, Whimbey E. Respiratory viral infections in immunocompetent and immunocompromised persons. Am J Med. 1997;102(3A):2-9.

12. Bowden RA. Respiratory virus infections after marrow transplant: the Fred Hutchinson Cancer Research Center experience. Am J Med. 1997;102(3A):27-30.

13. Ghosh S, Champlin RE, Englund J, Giralt SA, Rolston K, Raad I, et $a l$. Respiratory syncytial virus upper respiratory tract illnesses in adult blood and marrow transplant recipients: combination therapy with aerosolized ribavirin and intravenous immunoglobulin. Bone Marrow Transplant. 2000;25(7):751-5.

14. Shults RA, Baron S, Decker J, Deitchman SD, Connor JD. Health care worker exposure to aerosolized ribavirin: biological and air monitoring. J Occup Environ Med. 1996;38(3):257-63.

15. DeVincenzo JP, Hirsch RL, Fuentes RJ, Top FH Jr. Respiratory syncytial virus immune globulin treatment of lower respiratory tract infection in pediatric patients undergoing bone marrow transplantation - a compassionate use experience. Bone Marrow Transplant. 2000;25(2):161-5.

16. Khanna N, Widmer AF, Decker M, Steffen I, Halter J, Heim D, et al. Respiratory syncytial virus infection in patients with hematological diseases: single-center study and review of the literature. Clin Infect Dis. 2008;46(3):402-12.

17. Small TN, Casson A, Malak SF, Boulad F, Kiehn TE, Stiles J, et al. Respiratory syncytial virus infection following hematopoietic stem cell transplantation. Bone Marrow Transplant. 2002;29(4):321-7.

18. McCarthy AJ, Kingman HM, Kelly C, Taylor GS, Caul EO, Grier $\mathrm{D}$, et al. The outcome of 26 patients with respiratory syncytial virus infection following allogeneic stem cell transplantation. Bone Marrow Transplant. 1999;24(12):1315-22.

19. Crooks BN, Taylor CE, Turner AJ, Osman HK, Abinun M, Flood TJ, et al. Respiratory viral infections in primary immune deficiencies: significance and relevance to clinical outcome in a single BMT unit. Bone Marrow Transplant. 2000;26(10):1097-102.

20. Adams RH. Preemptive treatment of pediatric bone marrow transplant patients with asymptomatic respiratory syncytial virus infection with aerosolized ribavirin. Biol Blood Marrow Transplant. 2001;7 Suppl:16S-18S.

21. Khushalani NI, Bakri FG, Wentling D, Brown K, Mohr A, Anderson $\mathrm{B}$, et al. Respiratory syncytial virus infection in the late bone marrow transplant period: report of three cases and review. Bone Marrow Transplant. 2001;27(10):1071-3.

22. Machado CM, Boas LS, Mendes AV, Santos MF, da Rocha IF, Sturaro D, et al. Low mortality rates related to respiratory virus infections after bone marrow transplantation. Bone Marrow Transplant. 2003;31(8):695-700.

Avaliação: Editor e dois revisores externos

Conflito de interesse: sem conflito de interesse

Recebido: 01/02/2009

Aceito: 08/04/2009 